ARTICLE

\title{
Aberrant dynamics of cognitive control and motor circuits predict distinct restricted and repetitive behaviors in children with autism
}

\author{
Kaustubh Supekar (iD ${ }^{1 凶}$, Srikanth Ryali (iD ${ }^{1}$, Percy Mistry ${ }^{1} \&$ Vinod Menon (1) ${ }^{1,2,3 凶}$
}

Restricted and repetitive behaviors (RRBs) are a defining clinical feature of autism spectrum disorders (ASD). RRBs are highly heterogeneous with variable expression of circumscribed interests $(\mathrm{Cl})$, insistence of sameness (IS) and repetitive motor actions (RM), which are major impediments to effective functioning in individuals with ASD; yet, the neurobiological basis of $\mathrm{Cl}$, IS and RM is unknown. Here we evaluate a unified functional brain circuit model of RRBs and test the hypothesis that $\mathrm{Cl}$ and IS are associated with aberrant cognitive control circuit dynamics, whereas RM is associated with aberrant motor circuit dynamics. Using taskfree fMRI data from 96 children, we first demonstrate that time-varying cross-network interactions in cognitive control circuit are significantly reduced and inflexible in children with ASD, and predict $\mathrm{Cl}$ and IS symptoms, but not RM symptoms. Furthermore, we show that time-varying cross-network interactions in motor circuit are significantly greater in children with ASD, and predict RM symptoms, but not $\mathrm{Cl}$ or IS symptoms. We confirmed these results using cross-validation analyses. Moreover, we show that brain-clinical symptom relations are not detected with time-averaged functional connectivity analysis. Our findings provide neurobiological support for the validity of RRB subtypes and identify dissociable brain circuit dynamics as a candidate biomarker for a key clinical feature of ASD.

\footnotetext{
${ }^{1}$ Department of Psychiatry \& Behavioral Sciences, Stanford University, Stanford, CA, USA. ${ }^{2}$ Department of Neurology \& Neurological Sciences, Stanford University, Stanford, CA, USA. ${ }^{3}$ Stanford Neuroscience Institute, Stanford University, Stanford, CA, USA. ${ }^{凶}$ email: ksupekar@stanford.edu; menon@stanford.edu
} 
$\mathrm{R}$ estricted and repetitive behaviors (RRBs) have long been recognized as a core symptom of autism spectrum disorders (ASD) ${ }^{1}$. RRBs are the earliest detectable behavioral predictors of ASD and have adverse long-term consequences for acquisition of crucial life skills in individuals with the disorder ${ }^{2,3}$. Critically, recent changes to the Diagnostic and Statistical Manual of Mental Disorders have identified RRBs as central to understanding heterogeneity of clinical presentations in $\mathrm{ASD}^{4}$. However, RRBs remain a grossly understudied aspect of ASD research and the underlying brain circuits are unknown ${ }^{5,6}$.

RRBs include behaviors such as preoccupation with objects, ritualized patterns of behavior, highly restricted/fixated interests and stereotyped/repetitive motor (RM) movements ${ }^{1}$. Although RRBs were traditionally defined as a unitary construct ${ }^{5,7}$, there is growing evidence that RRBs are a heterogeneous construct that can be factored into three distinct phenotypic characteristics: circumscribed interests (CI), insistence on sameness (IS), and RM actions ${ }^{8,9}$. CI and IS include adherence to routines and restricted patterns of interest and are thought to be cognitive in nature ${ }^{10,11}$. In contrast, RM includes hand flapping, rocking, and head banging, and are likely to be primarily motoric in origin ${ }^{10,11}$. Recent evidence suggest that clinical phenotypic features may be more tightly linked to dynamical properties of functional brain circuits as they reflect complex nonlinear neural dynamics and fluctuations in internal mental states ${ }^{12-15}$. Whether CI, IS, and $\mathrm{RM}$ are associated with distinct dynamic brain circuit properties in ASD is currently unknown. Uncovering the brain circuit mechanisms underlying these heterogeneous RRB symptoms/ subtypes is important for a more precise understanding of the neurobiology of ASD and for further validating the distinctiveness of these individual phenotypic constructs.

Here we address critical gaps in our knowledge regarding heterogeneous expression of RRBs in childhood ASD using dynamic brain circuit analysis. We test the hypothesis that brain circuit dynamics underlying RRB symptoms can be dissociated, and specifically that, aberrant cognitive control circuit dynamics would underlie CI and IS symptoms whereas aberrant motor circuit dynamics would underlie RM symptoms. We characterize the dynamic properties of two distinct brain circuits: (i) a cognitive control circuit consisting of salience $(\mathrm{SN})^{16-18}$, central executive $(\mathrm{CEN})^{18-20}$, and default-mode $(\mathrm{DMN})^{21,22}$ network nodes that play a key role in salience detection, allocation of attentional resources, and flexible behavior and (ii) a motor circuit, consisting of cortical (cMN) and subcortical (sMN) motor network nodes important for implementing motor planning, control, and execution ${ }^{23}$ (Fig. 1a). We predicted that compared to TD children, children with ASD would show less flexible, aberrant time-varying brain circuit dynamics. In addition, we predicted that aberrant dynamics of the cognitive control circuit would be associated with CI and IS symptoms of RRB, but not $\mathrm{RM}$ symptoms and that aberrant dynamics of the motor circuit would be associated with RM symptoms of RRB, but not CI and IS symptoms. Finally, we predicted that, compared to static functional circuits, dynamic functional circuits would better distinguish and predict distinct RRB clinical symptoms.

\section{Results}

RRB subtypes derived from the ADI-R. Because age and IQ can influence RRB factor structure ${ }^{5}$, we first examined a cohort $(N=$ 126) of ASD participants in age and IQ matched to our imaging cohort. We performed principal component analysis (PCA) with varimax rotation on 9 Autism Diagnostic Interview-Revised (ADI-R) item-level scores ${ }^{24,25}$ (Supplementary Table 2). PCA found a three-component solution as the best solution, consistent with findings from previous studies albeit with different loadings ${ }^{8,9}$ (Supplementary Table 2). This three-component solution explained $54 \%$ of the variance, and each component included two to three items, as follows: (i) CI, which included, "Item 68: Circumscribed Interests" and "Item 76: Unusual Attachment to Objects", (ii), IS, which included "Item 67: Unusual Preoccupations" and "Item 70: Compulsions and Rituals", and (iii) RMB, which included "Item 69: Repetitive Use of Objects or Interests in Part of Objects", "Item 77: Hand and Finger Mannerisms" and "Item 78: Other Complex Mannerisms or Stereotyped Body Movements" (Supplementary Table 2). CI, IS, and RM scores derived from our sample were strongly correlated with scores derived using factor weights from a previously published study by Lam and colleagues ${ }^{9}$ (Spearman $\rho_{\mathrm{CI}}=0.88, p<$ $0.001, \rho_{\mathrm{IS}}=0.71, p<0.001, \rho_{\mathrm{RM}}=0.86, p<0.001$ ) (see Supplementary Results for details).

Temporal dynamics of cognitive control circuit in children with ASD and TD children. We next examined dynamic, timevarying, cross-network functional interactions in the cognitive control circuit and found four states (temporal clusters) in children with ASD and two in TD children (Fig. 2a, b), reflecting variation in cross-network interactions across time in both groups. Importantly, these results argue against assumptions of stationarity made in most previous functional connectivity studies in ASD.

We then compared the cognitive network interaction index (CNII) of dynamic brain states between the two groups. We computed CNII for each sliding window and averaged CNII for the windows corresponding to the same dynamic brain state. The mean CNII value, averaged across all states, was significantly lower in the ASD group, compared to the TD group $(p<0.0001, t$ $(94)=-4.07$, Cohen's $d=0.83$ ) (Fig. $2 c$ ), even after controlling for confounds such as age, sex, head motion, and IQ (Supplementary Table 3). These results demonstrate an intermittent lack of integration of the SN with the CEN and reduced decoupling of the SN from the DMN in children with ASD.

We next compared variability of dynamic time-varying crossnetwork interactions between the two groups and found that compared to TD children, children with ASD showed greater variability in CNII values across states, suggesting that crossnetwork interactions in the cognitive control circuit are more variable in ASD than TD group $(p<0.0001, t(94)=7.27$, Cohen's $d=1.48$ ) (Fig. 2c), even after controlling for confounds (Supplementary Table 4).

The aforementioned results were also observed for a different sliding window length (=40 s) as well as for a different sliding window shape (rectangular), demonstrating that the findings are robust against the length and shape of the sliding window.

Relation between temporal dynamics of cognitive control circuit and RRB subtypes in children with ASD. To investigate the extent to which atypical temporal dynamics of the cognitive control circuit is associated with severity of RRB subtypes in ASD, we examined the relationship between features of cognitive control circuit dynamics described above and ADI-R RRB factor scores.

Multivariate regression analysis revealed that mean and variability of CNII predicted CI scores $(F(2,45)=3.9, p<0.05)$ and IS scores $(F(2,45)=3.3, p<0.05)$ (see below). There was no significant relationship between mean and variability of CNII and $\mathrm{RM}(F(2,45)=0.48, p=0.63)$ (Supplementary Fig. 4$)$, emphasizing the specificity of the finding with CI and IS symptoms. To further examine the predictive ability of CNII, we performed a fivefold cross-validation analysis. Results from this analysis were consistent with the results from the original analysis ( $r$ (pred, 


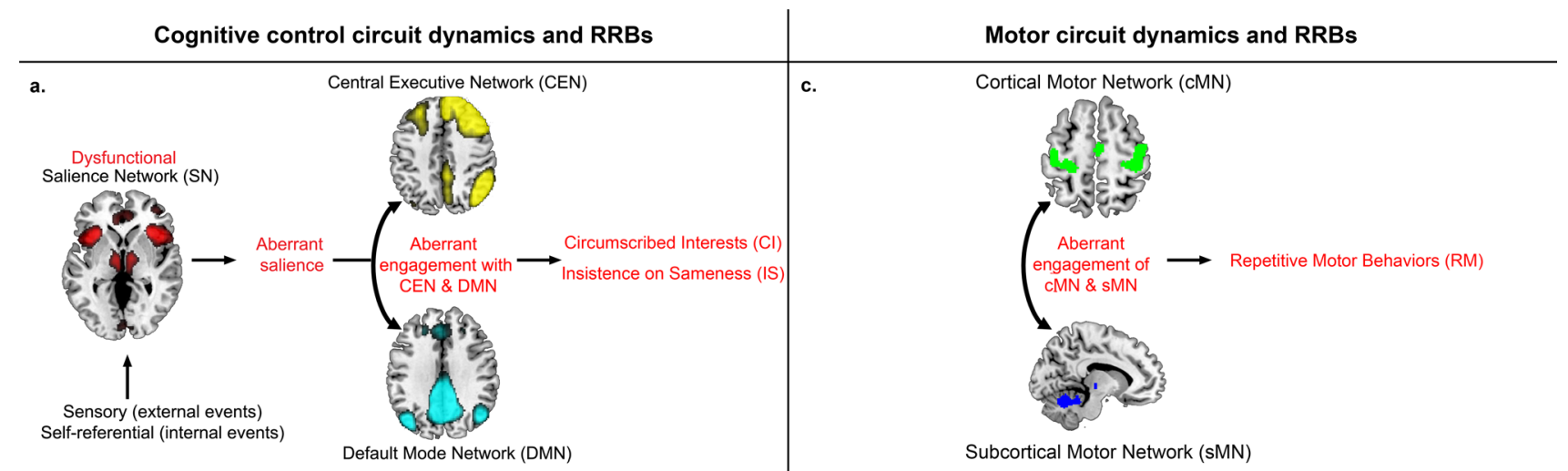

b.
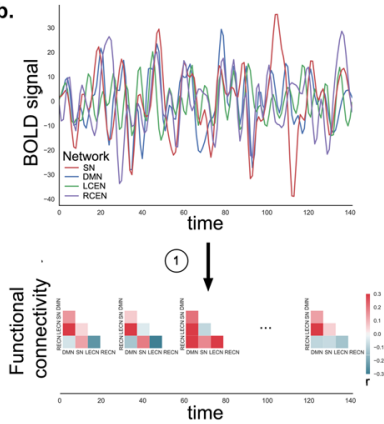

Cognitive circuit dynamics-based predictors of $\mathrm{Cl} \&$ IS symptoms severity $\uparrow(4)$

Quantitative measures of cognitive control circuit dynamics

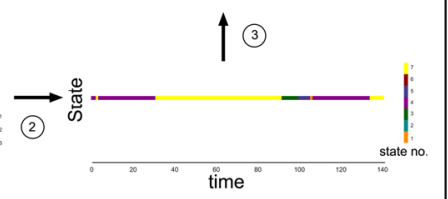

d.

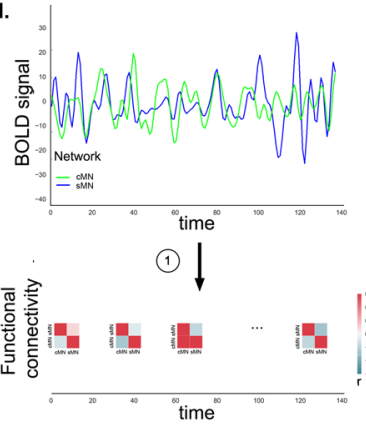

Motor circuit dynamics-based predictors of RM symptoms severity

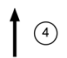

Quantitative measures of motor circuit dynamics

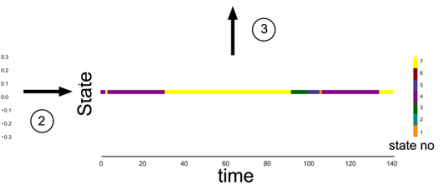

Fig. 1 Overall approach to determine the temporal dynamics of cognitive control and motor circuits and its relationship with Restricted and Repetitive Behaviors (RRBs). a Cognitive control circuit-based model of circumscribed interests (CI) and insistence on sameness (IS) symptoms. The model proposes that aberrant functional of organization of key fronto-parietal-opercular cognitive control circuit may contribute to the cognitive components of RRBs i.e., $\mathrm{Cl}$ and IS symptoms in children with ASD. Specifically, this model posits a key role for the salience network (SN) in aberrant mapping of internal and external salient events leading to altered dynamic temporal interactions with the central executive network (CEN), and the default mode network (DMN), resulting in $\mathrm{Cl}$ and IS. b Overall analysis pipeline for examining dynamic time-varying cross-network interactions within cognitive control circuit and their relationship with $\mathrm{Cl}$ and IS symptoms. Time-varying cross-network interaction was measured using a dynamic functional connectivity approach. (1) We estimated dynamic functional interactions between SN, CEN, and DMN using a sliding-window approach. (2) To identify distinct group-specific states associated with dynamic functional connectivity, we applied a group-wise k-means clustering on the time-series of correlation matrices in each group separately. (3) Brain state-specific network interaction index (NII) was used to characterize cross-network interaction in each dynamic brain state. $\mathrm{NII}$ for each state $\mathrm{k}$ by averaging NII across sliding-windows labeled as state $\mathrm{k}$. Cognitive NII (CNII) of a sliding window was computed as the difference in correlation between SN and CEN time series and correlation between SN and DMN. The correlation values were extracted from the covariance matrix associated with that sliding window. Mean and variability of time-varying CNII was calculated as average and standard deviation of CNII values across dynamics brain states respectively. (4) Linear regression analysis was used to examine the relation between dynamic time-varying cross-network interactions measure, including mean and variability of time-varying NII, and ADI-R derived RRB subtype symptom severity scores. c Motor circuit-based model of repetitive motor behavior (RM) symptoms. The model proposes that aberrant functional of organization of key cortical-subcortical motor circuit may contribute to the motoric components of RRBs i.e., RM symptoms in children with ASD. Specifically, this model posits a key role for altered dynamic temporal interactions between the cortical motor network (cMN), and the subcortical motor network (sMN), resulting in RM. d Overall analysis pipeline for examining dynamic time-varying cross-network interactions within motor circuit and their relationship with RM symptoms.

actual $)_{\mathrm{CI}}=0.29, p_{\mathrm{CI}}=0.01 ; r(\text { pred, actual })_{\mathrm{IS}}=0.33, p_{\mathrm{IS}}=0.01 ; r$ (pred, actual) $\left.)_{\mathrm{RM}}=-0.08, p_{\mathrm{RM}}=0.40\right)$. Similar results were observed with a tenfold cross-validation analysis (see Supplementary Materials for details), highlighting the stability of the findings. This finding was replicated with CI, IS, and RM scores computed using RRB factor weights previously published by Lam and colleagues ${ }^{9}$ (see Supplementary Results). Importantly, none of the aforementioned brain-behavior relations were detected with measures of static/time-averaged functional interactions in the cognitive control circuit, demonstrating the specificity of the findings to dynamical properties of the cognitive control circuit (see Supplementary Materials for details).

Temporal dynamics of motor circuit in children with ASD and TD children. We next examined dynamic cross-network functional interactions in the motor circuit and found two states (temporal clusters) in children with ASD and two in TD children
(Fig. 3a, b), reflecting variation in cross-network interactions across time in both groups, similar to the finding of cognitive control circuit.

We then compared the motor network interaction index (MNII) of dynamic brain states between the two groups. The mean MNII value was significantly higher in the ASD group, compared to the TD group $(p<0.05, t(94)=2.3$, Cohen's $d=$ 0.47) (Fig. 3c), even after controlling for confounds (Supplementary Table 5). These results demonstrate an intermittent increased coupling between the $\mathrm{cMN}$ and sMN in children with ASD.

We next compared variability of dynamic time-varying crossnetwork interactions between the two groups and found that variability in MNII values across states did not differ between the two groups ( $p=0.23, t(94)=-1.2$, Cohen's $d=0.25)$ (Fig. $3 c$ ).

The aforementioned results were also observed for a different sliding window length (=40 s) as well as for a different sliding window shape (rectangular), demonstrating that the findings are robust against the length and shape of the sliding window. 


\section{Cognitive control circuit dynamics}

a. Brain states

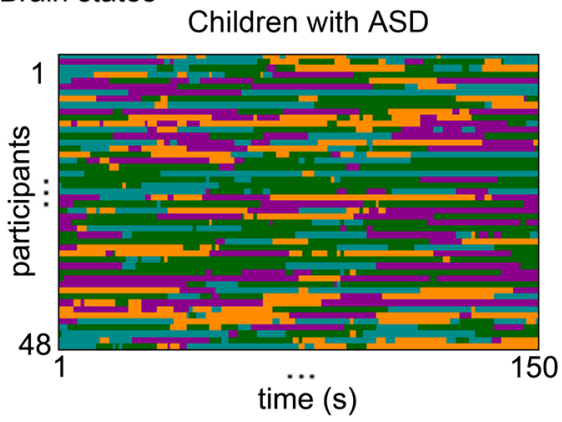

Typically-developing children

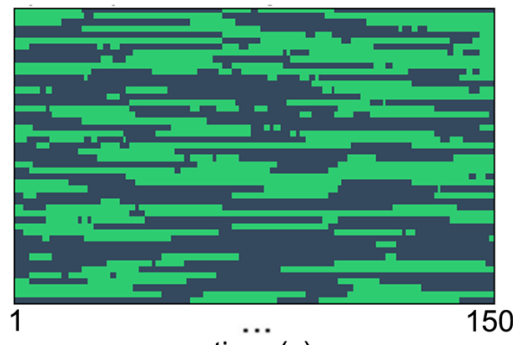

time (s)

b. Time-varying cognitive network interaction index (CNII)

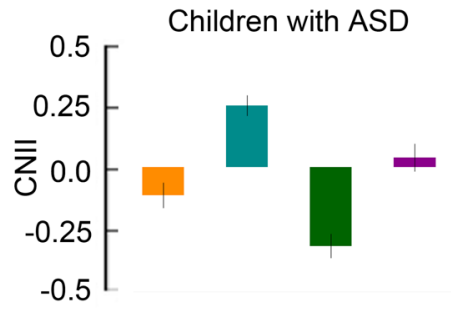

S1

$\ldots$

S4

S1

Typically-developing children

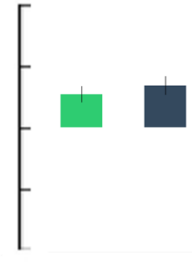

c. Mean and variability of CNII
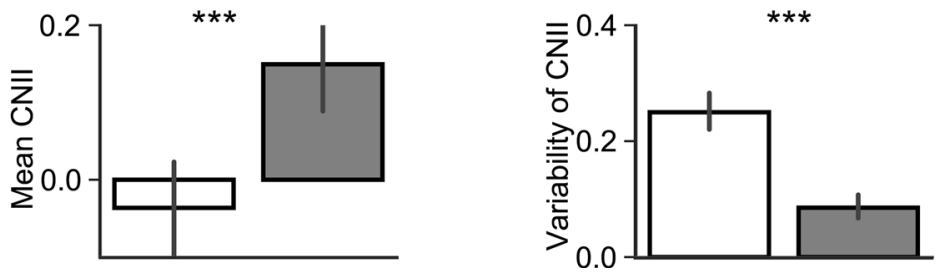

Fig. 2 Aberrant temporal dynamics of cognitive control circuit in children with ASD. a Children with ASD showed four states and TD children showed two states. Color codes distinct states in each participant. States 1, 2, 3, and 4 in children with ASD are represented by dark orange, dark cyan, dark green, and dark magenta, respectively; states 1 and 2 in TD children are represented by lime green and dark blue, respectively. b Cognitive NII (CNII) of dynamic brain states showed intermittently reduced cross-network interaction in children with ASD compared to TD children. c The temporal mean of dynamic cross-network interaction in the cognitive control circuit, assessed using mean of dynamic CNIIs across states, was significantly lower in children with ASD, compared to TD children. The temporal variability of dynamic cross-network interaction in the cognitive control circuit, assessed using standard deviation of dynamic CNIls across states, was significantly higher in children with ASD, compared to TD children. Two-sided two sample $t$-tests were used to compare mean and temporal variability of CNIls between children with ASD and TD children. Error bar shows standard error of mean. ${ }^{\star \star \star} p<0.001$.

Relation between temporal dynamics of motor circuit and RRB subtypes in children with ASD. To investigate the extent to which atypical temporal dynamics of the motor circuit is associated with severity of RRB subtypes in ASD, we examined the relationship between features of motor circuit dynamics described above and ADI-R RRB factor scores. Regression analysis revealed that mean of MNII predicted RM scores $(F(1,46)=5.2, p<0.05)$ (Fig. 4c). There was no significant relationship between mean MNII and CI $(F(1,46)=0.45, p=0.5)$ and IS $(F(1,46)=3.3, p$ $=0.07$ ) (Supplementary Fig. 4), emphasizing the specificity of this finding. To further examine the predictive ability of MNII, we performed fivefold cross-validation analysis. Results from this analysis were consistent with the results from the original analysis $(r \text { pred, actual })_{\mathrm{RM}}=0.29, p_{\mathrm{RM}}=0.005 ; r(\text { pred, actual })_{\mathrm{CI}}=-0.20$, $\left.p_{\mathrm{CI}}=0.53 ; r(\text { pred, actual })_{\mathrm{IS}}=0.13, p_{\mathrm{IS}}=0.09\right)$. Similar results were observed with a tenfold cross-validation analysis (see Supplementary Materials for details), highlighting the stability of the findings. This finding was replicated with RM, CS, and IS scores computed using RRB factor weights previously published by Lam and colleagues ${ }^{9}$ (see Supplementary Results). Importantly, the brain-behavior relation was not detected with measures of static/ time-averaged functional interactions in the motor circuit, demonstrating the specificity of the findings to dynamical properties of the motor circuit (see Supplementary Materials for details).

\section{Discussion}

The present study addresses a critical gap in our knowledge of brain circuits underlying CI, IS, and RM, three distinct symptom clusters that define RRB-a core clinical phenotype of ASD-in affected children. Using a systems neuroscience-based approach and dynamic functional circuits analysis we provide evidence that childhood ASD is characterized by aberrant dynamics of multiple 
Motor circuit dynamics

a. Brain states
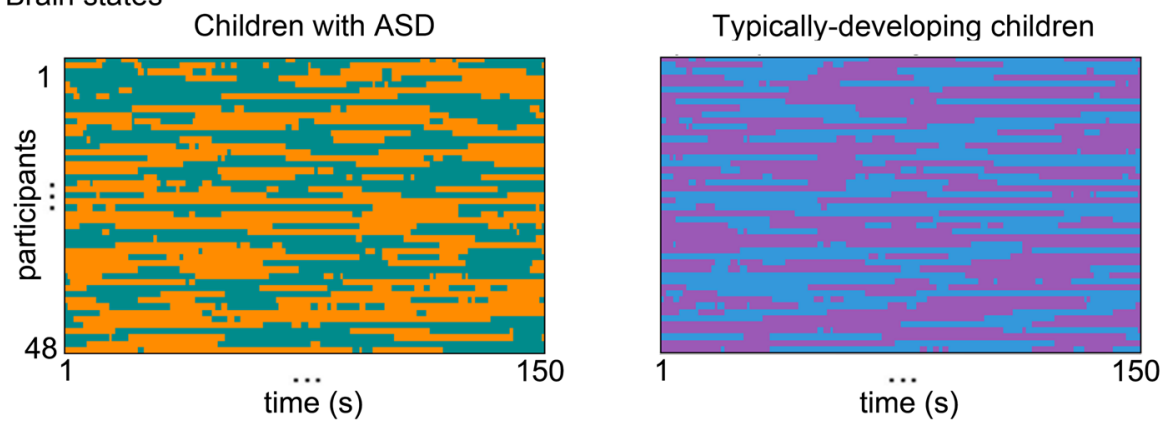

b. Time-varying motor network interaction index (MNII)
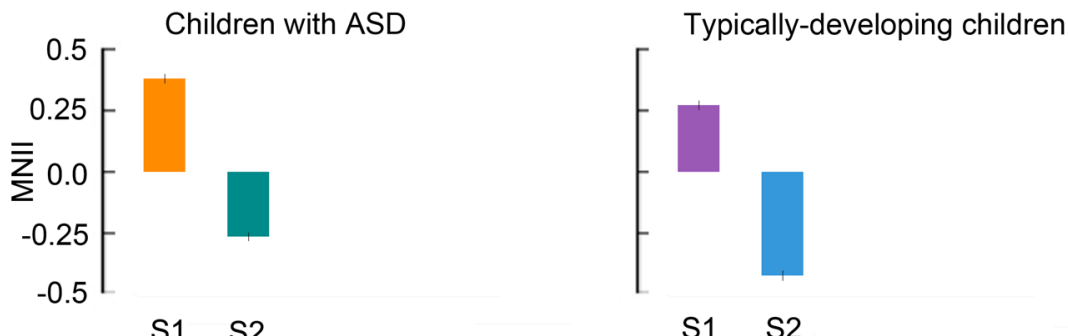

c. Mean and variability of MNII

$\square$ Children with ASD

$\square$ Typically-developing children
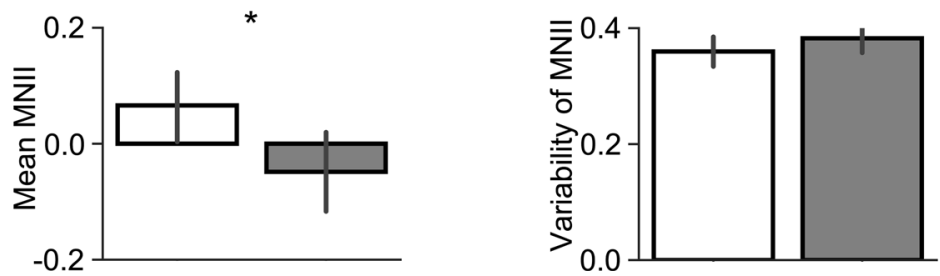

Fig. 3 Aberrant temporal dynamics of motor circuit in children with ASD. a Children with ASD and TD children showed two states. Color codes distinct states in each participant. States 1 and 2 in children with ASD are represented by dark orange and dark cyan, respectively; states 1 and 2 in TD children are represented by moderate violet and bright blue, respectively. b Motor NII (MNII) of dynamic brain states showed intermittently increased cross-network interaction in children with ASD compared to TD children. c The temporal mean of dynamic cross-network interaction in the motor circuit, assessed using mean of dynamic MNIIs across states, was significantly higher in children with ASD, compared to TD children. The temporal variability of dynamic crossnetwork interaction in the motor circuit, assessed using standard deviation of dynamic MNIIs across states, did not differ between children with ASD and TD children. Two-sided two sample $t$-tests were used to compare mean and temporal variability of MNIIs between children with ASD and TD children. Error bar shows standard error of mean. ${ }^{*} p<0.05$.

functional brain circuits, and crucially, that cognitive (CI, IS) and motoric (RM) RRB symptoms are associated with unique neurobiological signatures. Our findings demonstrate that dynamic properties of brain circuits can provide fundamental insights into mechanisms underlying heterogeneity of clinical symptoms in ASD.

Our dynamic connectivity analysis revealed that children with ASD have less flexible cognitive control circuit dynamics, characterized by brain states with impaired coupling of the SN with CEN and DMN, consistent with findings from a recent study that reported that adults with ASD show dominant neural states with aberrant functional interactions between SN and CEN and between $\mathrm{SN}$ and $\mathrm{DMN}^{26}$. Notably, we found that CI and IS symptoms of RRB were associated with the degree of inflexible interactions between the three key cognitive control networks: $\mathrm{SN}, \mathrm{CEN}$, and $\mathrm{DMN}^{16,27}$. Specifically, severity of $\mathrm{CI}$ and IS symptoms was associated with aberrant temporal engagement of the SN with the CEN and DMN. Notably, no such relation was found with RM symptoms pointing to the specificity of our findings with respect to cognitive inflexibility. Critically, no static time-averaged functional connectivity measures predicted CI or IS symptoms. These findings demonstrate that aberrant circuits dynamics associated with $\mathrm{SN}, \mathrm{CEN}$, and DMN carry clinically relevant neurobiological signatures of cognitive, but not motoric, components of RRB.

Cross-network interactions between the SN, CEN, and DMN play a key role in effectively responding to dynamic demands of changing environment ${ }^{16,27}$. In particular, interactions of the SN with the CEN and the DMN are thought to facilitate switching between externally-oriented attention and internally-oriented mental processes in response to salient events to guide flexible behavior $^{16,27}$. Our dynamic network analysis revealed that this 
a.

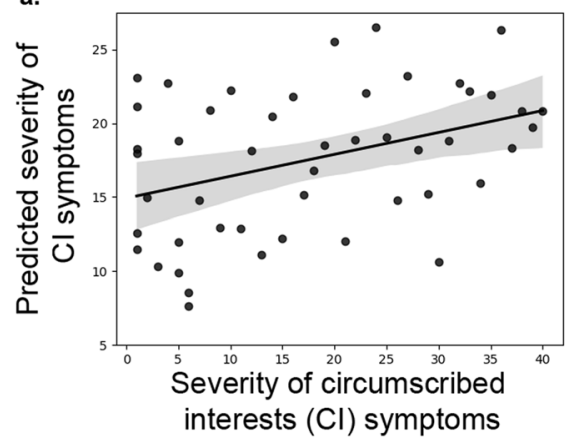

b.

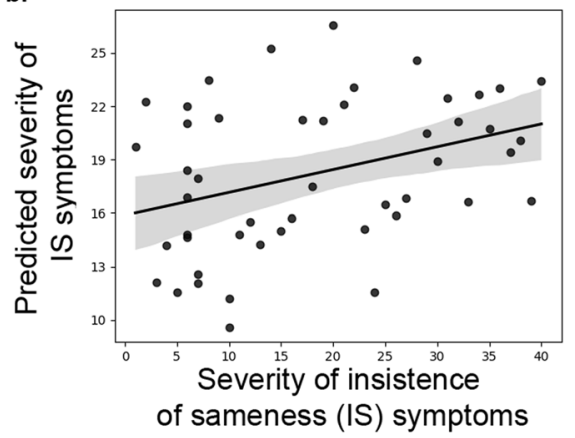

c.

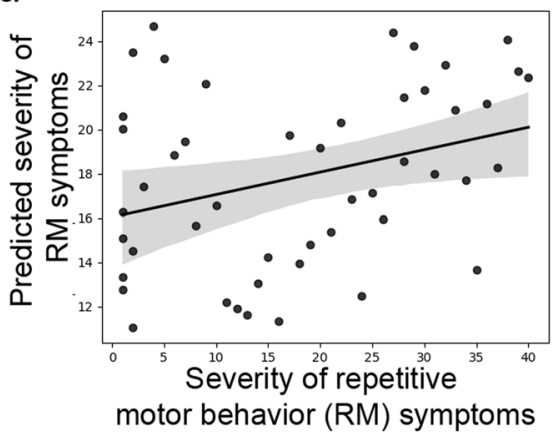

Fig. 4 Aberrant brain circuit dynamics in children with ASD predict RRB subtypes severity. Regression analysis revealed that temporal mean and variability of dynamic cross-network interactions in the cognitive control circuit predicted $\mathbf{a} \mathrm{Cl}$ and $\mathbf{b}$ IS symptoms, but not RM symptoms. $\mathbf{c}$ Regression analysis revealed that temporal mean of dynamic cross-network interactions in the motor circuit predicted RM symptoms, but not Cl or IS symptoms. Error band represent $95 \%$ confidence interval for the regression estimate. Cross-validation analyses confirmed these results.

switching is impaired in children with ASD, and that the degree of impairments predicts cognitive inflexibility. These results are consistent with and extend previous studies based on static timeaveraged measures demonstrating hyper-connectivity within the $\mathrm{SN}, \mathrm{CEN}$, and DMN in children with $\mathrm{ASD}^{28}$. Aberrant functioning of the anterior insula node of the $\mathrm{SN}$ in $\mathrm{ASD}^{29}$ may be a key mechanism contributing to inflexible circuits and behaviors given its key role as causal hub for switching between these networks ${ }^{16,27}$. Together, results suggest that reduced crossnetwork interactions in the cognitive control circuit contribute to core phenotypic features and inflexible behaviors such as intense focus, unusual attachment to objects of interest, and difficulty with changes in the environment, and provide support for a neurocognitive model of $\mathrm{RRB}^{7}$ in ASD based on dynamic circuit properties.

Analysis of motor circuit dynamics revealed a different pattern of association with specific RRB phenotypic features. Children with ASD had less flexible motor circuits characterized by stronger intermittent coupling between sMN and cMN. Moreover, we found a strong association between aberrant motor circuit dynamics and RM, but not CI and IS, pointing to the specificity of our findings with respect to motor symptoms. Our results highlight a tight link between $s M N-c M N$ dynamics and RM symptoms observed in children with ASD.

The sMN and cMN nodes including the cerebellum, motor, and premotor regions are critical for motor control and execution ${ }^{23}$, and have been shown to have structural abnormalities in individuals with $\mathrm{ASD}^{30,31}$. Our results suggest reduced differentiation of these motor networks ${ }^{32}$ can lead to more rigidity in motor behaviors. We previously suggested that intrinsically hyper-connected circuits may make it more difficult to modulate connectivity in response to task demands, thereby resulting in task-related under-connectivity compared to the baseline state ${ }^{33}$. Consistent with this proposal, a previous study reported reduced static connectivity between the subcortical and cortical motor regions during a finger sequencing task in individuals with autism ${ }^{34}$. Thus, we hypothesize that the propensity of children with ASD to remain in brain states in which sMN and cMN nodes are intrinsically hyper-connected, potentially due to structural deficits in the fronto-thalamo-cerebellum pathway $^{32}$, could lead to inflexible motor control ${ }^{34,35}$ and RM behaviors that are characteristic of the disorder ${ }^{1}$.

To address growing concerns about reproducibility of neuroscientific findings ${ }^{36}$, we leveraged our sample and conducted cross-validation analyses following procedures typically used in machine learning. Cross-validation is a powerful approach for validating research findings, and its use for demonstrating generalization and reproducibility has been advocated in psychiatry, psychology, and many other disciplines ${ }^{37,38}$. The results of these analyses were consistent with our original results, demonstrating the robustness of our findings. Finally, findings were replicated with RRB measures derived from a previously published factor structure ${ }^{9}$.

In conclusion, the present study is the first to demonstrate that CI, IS, and RM behaviors, the three phenotypic components of $\mathrm{RRB}$, are associated with distinct features of brain circuit dynamics in childhood ASD. In contrast, such brain-clinical symptom associations were not observed with static timeaveraged connectivity measures. Future work is needed to determine whether these findings, (i) observed in a predominantly male sample consistent with extant neuroimaging studies of ASD, extend to females with ASD, (ii) using 6 min fMRI scans, extend to longer scans, and (iii) using ADI-R, extend to other RRB scales such as Repetitive Behavioral Scale-Revised (RBS-R). Our findings of inflexible functional circuits provide a dynamic brain circuit model of RRB subtypes. Identification of unique neurobiological signatures underlying these symptoms may assist in the development of symptom-based biomarkers and treatments, including TMS that target cognitive control and motor circuits deficits identified here, in affected individuals. Importantly, our findings provide, to the best of our knowledge, novel neurobiological support for the validity of RRB subtypes. More generally, the parsimonious predictive framework and computational methods developed here may prove widely useful for better characterizing clinical heterogeneity in other psychiatric disorders based on systems-neuroscience based models of brain circuit dynamics.

\section{Methods}

Participants. The clinical part of the study wherein we examined the structure of the RRB symptoms included 126 children with ASD (112 males, 14 females; age: $10.0 \pm 1.6$ years; IQ: $110 \pm 16)$. The imaging part of the study included: 48 children with ASD (41 males, 7 females; age: $10.9 \pm 1.9$ years; IQ: $115 \pm 16)$ and 48 age- and gender-matched TD children (41 males, 7 females; age: $10.9 \pm 1.7$ years; IQ: $118 \pm 11$ ) (Supplementary Table 1 and Supplementary Fig. 1). Informed written consent was obtained from the legal guardian of each child and the study protocol was approved by the Stanford University Institutional Review Board. The ASD diagnosis procedure and the subject inclusion/exclusion criteria are described in detail in Supplementary Materials.

We also conducted a search (Supplementary Fig. 2) of publicly-available opensource ASD datasets including ABIDE (http://fcon_1000.projects.nitrc.org/indi/ abide/) and the NIMH Data Archive (https://nda.nih.gov/) and found that none of these data contain item-level ADI- $\mathrm{R}^{24,25}$ scores, crucial phenotypic data relevant to our study, highlighting the uniqueness of our data.

Clinical measures and analysis. Similar to previous studies, we used ADI-R to assess RRBs in each child with $\mathrm{ASD}^{8,9}$. To determine the factor structure of the RRB symptom domain as measured by the ADI-R, we performed a PCA with varimax rotation on ADI-R items, using Matlab R2018v5.0. In line with procedures described 
in previous studies ${ }^{8,9}$, nine ADI-R items which assess RRBs were included in the analysis (Supplementary Table 2). "Current" behavior ratings were used and scores of 6,7 , and 8 were converted to 0 . The number of components extracted in PCA was determined using a combination of eigenvalues above 1 and scree plot.

Imaging data acquisition. Each participant underwent a 6-min resting-state fMRI scan and a T1-weighted structural imaging scan on a $3 \mathrm{~T}$ GE Signa scanner in the same session. Participants were instructed to stay awake, keep their eyes closed, and try not to move for the duration of the 6-min scan. Imaging data acquisition protocol and parameters are described in detail in Supplementary Materials.

Imaging data analysis. Overall imaging data analysis pipeline is illustrated in Fig. 1. Imaging data were preprocessed ${ }^{14}$ (see Supplementary Materials for details) and the preprocessed resting-state fMRI data were entered into a group independent component analysis (ICA) to identify SN, left CEN, right CEN, DMN, cMN, and sMN.

\section{Dynamic functional brain circuit analysis}

Cognitive control circuit. We applied dynamic functional connectivity analysis on the SN, CEN, and DMN timeseries data in each group (see Supplementary Materials for details and Supplementary Fig. 3). Briefly, we first estimated dynamic functional interactions between SN, CEN, and DMN using an exponentially decaying sliding window. Second, we identified distinct group-specific states associated with dynamic functional connectivity, using group-wise k-means clustering. The optimal number of clusters, i.e., states, was determined using maximal silhouette across multiple iterations ${ }^{39}$. Because our goal was to investigate whether dynamic temporal properties differed between the two groups (children with ASD and TD children), we allowed the number of clusters to differ between the groups, instead of keeping them exactly the same ${ }^{14}$. Third, we characterized cross-network interaction in each dynamic brain state, using brain state-specific CNII. CNII measures cross-network interactions among the three networks based on the hypothesized role of the SN in switching interactions with the CEN and $\mathrm{DMN}^{16,17}$. CNII has the advantage of capturing interactions simultaneously among all three networks. Specifically, CNII was computed as the difference in correlation between SN and CEN time series and correlation between SN and DMN. CNII thus captures the extent to which SN temporally engages with CEN and dissociates itself from $\mathrm{DMN}^{21,17}$. We computed CNII for each sliding window and the (i) mean and (ii) variability (measured by standard deviation) of time-varying CNII across all the dynamic brain states for each participant. We then examined the difference between the mean and variability of time-varying CNII between the two groups using two sample $t$-tests. Non-parametric linear regression was used to test associations between time-varying functional connectivity metrics of the cognitive control circuit and RRB subtypes. To further examine the predictive ability of cognitive control circuit dynamics and assess reproducibility, we leveraged our sample and conducted fivefold and tenfold cross-validation analyses $37,38,40$ (see Supplementary Materials for details).

Motor circuit. We applied dynamic functional connectivity analysis procedures on the cMN and sMN timeseries data in each group (see Supplementary Materials for details and Supplementary Fig. 3). Briefly, we first estimated dynamic functional interactions between $\mathrm{cMN}$ and sMN using an exponentially decaying sliding window. Second, we identified distinct group-specific states associated with dynamic functional connectivity, using group-wise k-means clustering. Third, we characterized cross-network interaction in each dynamic brain state, using brain state-specific MNII. MNII measures cross-network interactions among the two networks involved in motor function and was computed as the correlation between $\mathrm{cMN}$ and sMN time series. MNII thus captures the extent to which cMN temporally engages with sMN. We computed MNII for each sliding window and the (i) mean and (ii) variability (measured by standard deviations) of time-varying MNII across all the sliding windows for each participant. We then examined the difference between the mean and variability of time-varying MNII between the two groups using two sample $t$-tests. Non-parametric linear regression was used to test associations between time-varying functional connectivity metrics of the motor circuit and RRB subtypes. To further examine the predictive ability of motor circuit dynamics, we used fivefold and tenfold cross-validation analyses (see Supplementary Materials for details).

Reporting summary. Further information on research design is available in the Nature Research Reporting Summary linked to this article.

\section{Data availability}

All data that support the findings of this study are available from the corresponding authors upon reasonable request.

\section{Code availability}

Data were analyzed using Matlab 9.5 (R2018b) and SPM8. Data analysis scripts will be made available via GitHub upon publication.
Received: 29 June 2020; Accepted: 19 May 2021;

Published online: 10 June 2021

\section{References}

1. Kanner, L. Autistic disturbances of affective contact. Nerv. Child 2, 217-250 (1943).

2. Wolff, J. J. et al. Longitudinal patterns of repetitive behavior in toddlers with autism. J. Child Psychol. Psychiatry 55, 945-953 (2014).

3. Morgan, L., Wetherby, A. M. \& Barber, A. Repetitive and stereotyped movements in children with autism spectrum disorders late in the second year of life. J. Child Psychol. Psychiatry 49, 826-837 (2008).

4. APA. Diagnostic and statistical manual of mental disorders: DSM-5 (American Psychiatric Association, 2013).

5. Leekam, S. R., Prior, M. R. \& Uljarevic, M. Restricted and repetitive behaviors in autism spectrum disorders: a review of research in the last decade. Psychol. Bull. 137, 562-593 (2011).

6. Wilkes, B. J. \& Lewis, M. H. The neural circuitry of restricted repetitive behavior: magnetic resonance imaging in neurodevelopmental disorders and animal models. Neurosci. Biobehav. Rev. https://doi.org/10.1016/j. neubiorev.2018.05.022 (2018)

7. Traynor, J. \& Hall, G. Structural and functional neuroimaging of restricted and repetitive behavior in autism spectrum disorder. J. Intellect. Disabil. Diagn. Treat. 3, 21-34 (2015).

8. Honey, E., McConachie, H., Randle, V., Shearer, H. \& Couteur, A. S. One-year change in repetitive behaviours in young children with communication disorders including autism. J. Autism Dev. Disord. 38, 1439-1450 (2008).

9. Lam, K. S., Bodfish, J. W. \& Piven, J. Evidence for three subtypes of repetitive behavior in autism that differ in familiality and association with other symptoms. J. Child Psychol. Psychiatry 49, 1193-1200 (2008).

10. Lewis, M. H., Tanimura, Y., Lee, L. W. \& Bodfish, J. W. Animal models of restricted repetitive behavior in autism. Behav. Brain Res. 176, 66-74 (2007).

11. Turner, M. Annotation: repetitive behaviour in autism: a review of psychological research. J. Child Psychol. Psychiatry 40, 839-849 (1999).

12. Calhoun, V. D., Miller, R., Pearlson, G. \& Adali, T. The chronnectome: timevarying connectivity networks as the next frontier in fMRI data discovery. Neuron 84, 262-274 (2014).

13. Chen, T., Cai, W., Ryali, S., Supekar, K. \& Menon, V. Distinct global brain dynamics and spatiotemporal organization of the salience network. PLoS Biol. 14, e1002469 (2016).

14. Cai, W., Chen, T., Szegletes, L., Supekar, K. \& Menon, V. Aberrant timevarying cross-network interactions in children with attention-deficit/ hyperactivity disorder and the relation to attention deficits. Biol. Psychiatry Cogn. Neurosci. Neuroimaging 3, 263-273 (2018).

15. Supekar, K., Cai, W., Krishnadas, R., Palaniyappan, L. \& Menon, V. Dysregulated brain dynamics in a triple-network saliency model of schizophrenia and its relation to psychosis. Biol. Psychiatry 85, 60-69 (2019).

16. Menon, V. \& Uddin, L. Q. Saliency, switching, attention and control: a network model of insula function. Brain Struct. Funct. 214, 655-667 (2010).

17. Menon, V. in Brain Mapping: An Encyclopedic Reference (ed. Arthur, W. Toga) 449-459 (Academic Press: Elsevier, 2015).

18. Seeley, W. et al. Dissociable intrinsic connectivity networks for salience processing and executive control. J. Neurosci. 27, 2349-2356 (2007).

19. Dosenbach, N. et al. Distinct brain networks for adaptive and stable task control in humans. Proc. Natl Acad. Sci. USA 104, 11073-11078 (2007).

20. Dosenbach, N. U., Fair, D. A., Cohen, A. L., Schlaggar, B. L. \& Petersen, S. E. A dual-networks architecture of top-down control. Trends Cogn. Sci. 12, 99-105 (2008).

21. Greicius, M. D., Krasnow, B., Reiss, A. L. \& Menon, V. Functional connectivity in the resting brain: a network analysis of the default mode hypothesis. Proc. Natl Acad. Sci. USA 100, 253-258 (2003).

22. Fox, M. D. \& Raichle, M. E. Spontaneous fluctuations in brain activity observed with functional magnetic resonance imaging. Nat. Rev. Neurosci. 8 , 700-711 (2007).

23. Bressler, S. L. \& Menon, V. Large-scale brain networks in cognition: emerging methods and principles. Trends Cogn. Sci. 14, 277-290 (2010).

24. Le Couteur, A. et al. Autism diagnostic interview: a standardized investigatorbased instrument. J. Autism Dev. Disord. 19, 363-387 (1989).

25. Lord, C., Rutter, M. \& Le Couteur, A. Autism diagnostic interview-revised: a revised version of a diagnostic interview for caregivers of individuals with possible pervasive developmental disorders. J. Autism Dev. Disord. 24, 659-685 (1994)

26. Watanabe, T. \& Rees, G. Brain network dynamics in high-functioning individuals with autism. Nat. Commun. 8, 16048 (2017). 
27. Sridharan, D., Levitin, D. J. \& Menon, V. A critical role for the right frontoinsular cortex in switching between central-executive and default-mode networks. Proc. Natl Acad. Sci. USA 105, 12569-12574 (2008).

28. Uddin, L. Q. et al. Salience network-based classification and prediction of symptom severity in children with autism. JAMA Psychiatry 70, 869-879 (2013).

29. Uddin, L. Q. \& Menon, V. The anterior insula in autism: under-connected and under-examined. Neurosci. Biobehav. Rev. 33, 1198-1203 (2009).

30. DeRamus, T. P. \& Kana, R. K. Anatomical likelihood estimation meta-analysis of grey and white matter anomalies in autism spectrum disorders. Neuroimage Clin. 7, 525-536 (2015).

31. Mahajan, R., Dirlikov, B., Crocetti, D. \& Mostofsky, S. H. Motor circuit anatomy in children with autism spectrum disorder with or without attention deficit hyperactivity disorder. Autism Res. https://doi.org/10.1002/aur.1497 (2015).

32. Carper, R. A., Solders, S., Treiber, J. M., Fishman, I. \& Muller, R. A. Corticospinal tract anatomy and functional connectivity of primary motor cortex in autism. J. Am. Acad. Child Adolesc. Psychiatry 54, 859-867 (2015).

33. Uddin, L. Q. et al. Brain state differentiation and behavioral inflexibility in autism. Cereb. Cortex 25, 4740-4747 (2015).

34. Mostofsky, S. H. et al. Decreased connectivity and cerebellar activity in autism during motor task performance. Brain 132, 2413-2425 (2009).

35. Dziuk, M. A. et al. Dyspraxia in autism: association with motor, social, and communicative deficits. Dev. Med. Child Neurol. 49, 734-739 (2007).

36. Button, K. S. et al. Power failure: why small sample size undermines the reliability of neuroscience. Nat. Rev. Neurosci. 14, 365-376 (2013).

37. Koul, A., Becchio, C. \& Cavallo, A. Cross-validation approaches for replicability in psychology. Front. Psychol. 9, 1117 (2018).

38. Bzdok, D. \& Meyer-Lindenberg, A. Machine learning for precision psychiatry: opportunities and challenges. Biol. Psychiatry Cogn. Neurosci. Neuroimaging 3 , 223-230 (2018).

39. Bellec, P., Rosa-Neto, P., Lyttelton, O. C., Benali, H. \& Evans, A. C. Multi-level bootstrap analysis of stable clusters in resting-state fMRI. Neuroimage 51, 1126-1139 (2010).

40. Hastie, T., Tibshirani, R. \& Friedman, J. H. The Elements of Statistical Learning: Data Mining, Inference, and Prediction (Springer, 2009).

\section{Acknowledgements}

We greatly appreciate the contributions of participating children and their parents without which this work would not be possible. We thank Drs. Jennifer Phillips, Hyesang Chang for valuable feedback, and Samantha Mitsven for collating the item-wise ADI-R scores. This research was supported by grants from the National Institutes of Health
(MH084164, EB022907, and MH121069) to V.M., and by a NARSAD Young Investigator Award, a Stanford Innovator Award, and a grant from the Stanford Maternal and Child Health Research Institute through the Transdisciplinary Initiatives Program and the Taube Maternal and Child Health Research Fund to K.S.

\section{Author contributions}

K.S. and V.M. conceived and designed the study; K.S. analyzed the data; S.R. and P.M. contributed methods; K.S. and V.M. wrote the paper.

\section{Competing interests}

The authors declare no competing interests.

\section{Additional information}

Supplementary information The online version contains supplementary material available at https://doi.org/10.1038/s41467-021-23822-5.

Correspondence and requests for materials should be addressed to K.S. or V.M.

Peer review information Nature Communications thanks Francisco Castellanos and the other, anonymous, reviewer(s) for their contribution to the peer review of this work.

Reprints and permission information is available at http://www.nature.com/reprints

Publisher's note Springer Nature remains neutral with regard to jurisdictional claims in published maps and institutional affiliations.

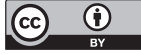

Open Access This article is licensed under a Creative Commons Attribution 4.0 International License, which permits use, sharing, adaptation, distribution and reproduction in any medium or format, as long as you give appropriate credit to the original author(s) and the source, provide a link to the Creative Commons license, and indicate if changes were made. The images or other third party material in this article are included in the article's Creative Commons license, unless indicated otherwise in a credit line to the material. If material is not included in the article's Creative Commons license and your intended use is not permitted by statutory regulation or exceeds the permitted use, you will need to obtain permission directly from the copyright holder. To view a copy of this license, visit http://creativecommons.org/ licenses/by/4.0/

(C) The Author(s) 2021 